\title{
Exploring New Ways of eTandem and Tele- collaboration Through the WebRTC Protocol: Students' Engagement and Perceptions
}

\author{
https://doi.org/10.3991/ijet.v14i05.9612 \\ Alberto Andujar ( $\left.{ }^{\bowtie}\right)$, Cristóbal Medina-López \\ University of Almería, Almería Spain \\ alberto.andujar@ual.es
}

\begin{abstract}
The present research investigates students' degree of engagement and perceptions in a telecollaboration project which incorporates the use of the WebRTC (Web Real-time Communication) protocol and Peer-to-Peer (P2P) communication to develop a virtual exchange in a second language environment. WebRTC is an open source technology that simplifies the telecommunication process thanks to its inherent characteristics such as P2P connectivity, no plugins installation, scalability and accessibility. The study involved 32 participants from two different universities who participated in a tele-tandem exchange over a period of 4 months. Task design consisted of information exchange and information gap activities that were given to students in order to foster the interaction. A mixed methods approach was used and students' engagement was measured through the use of a log, a pre-existing knowledge survey and an engagement scale. An additional survey analyzing students' perceptions was also given to students at the end of the project. Inferential statistics showed significant results in terms of emotional, behavioral and cognitive engagement. Furthermore, WebRTC earned a high acceptance among students participating in the virtual exchange and advantages and pitfalls of using this technology were observed throughout the project.
\end{abstract}

Keywords-Tele-collaboration, eTandem, WebRTC, P2P, Virtual Exchange, Tele-tandem, eLearning

\section{Introduction}

The need for teachers to adopt the latest technological advances has become a constant in today's education in order to keep pace with the latest gadgets and devices students use inside and out of the classroom environment. In this respect, distance learning constituted a shift from the traditional classroom, providing access to contents at different times and places, thus breaking spatial and temporal lines.

From the perspective of the teacher, the change in the paradigm has created a constant need for finding the latest technological applications that could potentially influence students' development. In terms of language learning, videoconferencing and 
telecollaboration have been widely investigated due to the possibilities that both types of online communication offer for language practice. In this sense, telecollaboration has become a virtual learning environment (VLE) where students interact and group work from different places through the use of digital or online communication tools [1]. Higher institutions and practitioners have shaped this process depending on the available systems to connect peers and classrooms, exploring its possibilities in a variety of settings. Specifically, in the case of language learning, the characteristics of telecollaboration have been exploited for language exchange, social interaction, communication and learning among others. Within the different modalities of telecollaboration in the second language field, Belz \& Thorne [2] as well as PérezHernández [3] refer to tele-tandem when students take turns to exchange the role of the native speaker, benefiting from the interaction in the target language. The growing number of articles dealing with telecollaboration has been pointed out by experts in the field such as Dooly and O'Dowd [4] who stated that one of the main reasons for this growth was the escalation of easily accessible technology in the classroom. In this vein, different platforms and software have been used to achieve telecollaboration through internet connectivity, from an early use of emails to achieve an asynchronous bilingual exchange with native speakers [5] to the use of Skype platform [6], [7] providing videoconferencing and Peer-to-Peer (P2P) communication.

During this search for the best application or system to undertake cultural exchanges, carry out collaborative projects, or simply give a lesson, different platforms have emerged such as Adobe Connect. This application has led the market by offering a stable platform where students are able to attend classes or communicate with the teacher via chat or through the webcam. Nevertheless, leading videoconferencing applications require premium memberships, and in many countries and institutions it is still not possible to afford the expenses resulting from subscribing to these platforms. With the release of Web real-time communication (WebRTC), teachers and students no longer have the need to download and install a screen-casting application or software [8], avoiding the consequences of these processes as could be students failing in accessing the virtual exchange platform, or having to afford the cost of the software. WebRTC technology offers free P2P communication through the browser, thus no preinstalling is needed and merely with a link, students are able to communicate with the teacher through the web. This technology offers the possibility to connect browsers through a P2P protocol in which built-in components such as audio, video or image-sharing allow telecollaboration processes. Furthermore, as of February $2018,80 \%$ of the existing browsers have fully or partially adopted this technology as could be the case of Google Chrome, Safari, Mozilla Firefox, Microsoft Edge or Opera [9]. In addition, WebRTC is supported by the leading browsers not only on computers but also on mobile devices running both Android and iOS, conferring a large set of devices with a tremendous potential for $\mathrm{P} 2 \mathrm{P}$ communication.

Thus, this investigation seeks to cover the literature gap as regards the use of this protocol within the framework of a bilingual exchange, analyzing students' engagement and perceptions. The web programme Jitsi, whose online version is based on WebRTC and P2P communication, was used as it offered possibilities for videoconferencing, screen-sharing, document sharing as well as chat functionalities, thus al- 
most the same characteristics as any other premium videoconferencing system. Nevertheless, as this technology is based on a P2P connection, information does not go through a server, improving the speed of the data exchange and avoiding delays in the conversation. In this sense, thanks to the accessibility, speed and characteristics of the system, the WebRTC protocol could potentially improve and enrich language exchange as well as possibilities for general instruction.

\section{System Overview}

Nowadays, a Client-Server (CS) model is the dominant approach on most of the communication models used by the most popular applications for video transmission, including chat and videoconferencing apps. In this approach, the stream is uploaded to a server which broadcasts to a client or a set of clients, and its popularity has to do with its simplicity, and of course, with the fact that the content provider has total control over the content transmitted. However, a CS approach has some disadvantages from the user's point of view such as scalability issues, privacy issues, and potential communication delays. In this context, P2P architectures can help solve these issues because, by definition, P2P is more scalable as the source of the media sends less quantity of data, peers being responsible for resending the media as many times as needed, taking advantage of their upload bandwidth. In addition, as there is no need to use a server, $\mathrm{P} 2 \mathrm{P}$ becomes a more private solution as the flow of data does not go through a third-party server, but from peer to peer directly. This fact reduces the potential delays that an intermediary server could cause.

Figure 1 shows the differences between the communication process in a CS model and a P2P model for an eTandem application. In the first one, all the data sent and received by Student 1 is going through a third party server which resends this information to Student 2 (and vice versa). However, in a P2P model, all the information goes from Student 1 to Student 2 directly, without the need for a third party server.

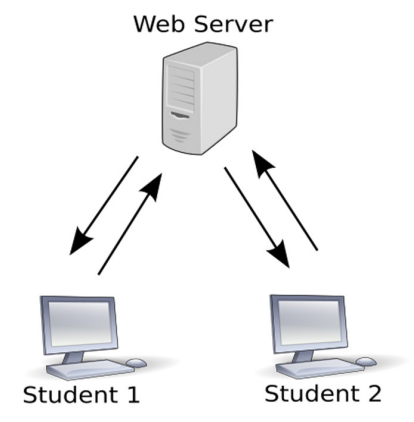

Client - Server Model

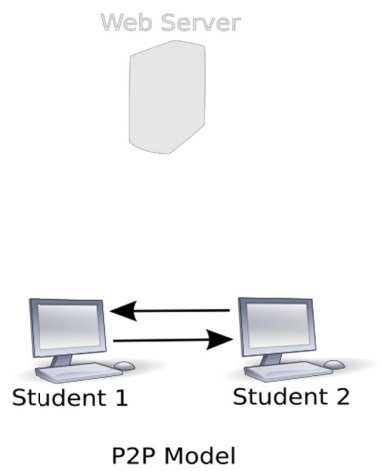

P2P Model

Fig. 1. Communication scheme in a Client-Server model vs communication scheme in a P2P Model. 
2.1 Some of the advantages of using WebRTC over other communication technologies

- Peer to peer communication: Connections between peers are carried out following a P2P model instead of a client-server model. It makes sure that the information is private because it does not go through a centralized server, improving the transmission speed.

- Web-based technology: The user only has to open a new tab in their favorite Web browser to get all benefits of WebRTC, including access to the webcam and microphone as well as $\mathrm{P} 2 \mathrm{P}$ communication facilities.

- No plugins need to be installed. WebRTC is supported by default and it is not necessary to install additional software at the browser or operating system level.

- Abstraction of NATing and Firewall issues: It supports proxies and includes a solution for dealing with NATs and Firewall problems in a transparent way for users by using ICE, STUN and TURN technologies.

- Free and Open Source: By using a free tool, it is possible to reduce costs for the company or institution, especially if an organization does not have a huge budget to access. Moreover, since the code of the software is available, customised applications can be built by organisations in a cheaper and faster way.

- Most of the browsers support this technology: Since its appearance, it has caught the attention of several working groups and companies which work to improve the support of the protocol and its API for several browsers. Today, WebRTC is in the final stage of the standardization process.

\section{Literature Review}

There is a great number of studies using these web-communication tools for different educational fields and at different stages of education. In this vein, different studies have explored the possibilities videoconferencing systems offer for teaching and learning through comparing the existing applications in the market. This was the case of Karabulut and Correia [10] who analysed the use of Elluminate, Adobe Connect, Skype and Ivisit, or Schullo, Hilbelink, Venable \& Barron [11] who focused on Elluminate and Adobe Connect by comparing the differences among the platforms as well as its characteristics. Recent investigation regarding teaching and learning through videoconferencing tools have made use of some of the most used applications such as Adobe Connect [12], [13], [14] or skype [15], [16] to foster students' development and promote online and distance teaching and learning.

In terms of language learning similar platforms and web applications have been used to carry out telecollaboration studies where different aspects of second language learners were investigated. In this sense, linguistic gains, intercultural competence and the characteristics of the conversations taking place during language exchange were observed. Studies using telecollaboration tools such as Skype agreed on the fact that these online platforms have become a fertile ground for language exchange as claimed by Tian and Wang [6], who focused on the improvements learners perceived through the use of eTandem via Skype. In a similar vein, further studies reporting linguistic 
gains through the use of skype pointed out its potential to develop students' listening comprehension [17] as well as motivation [18], [19] and oral development [16], [20], [21], [22]. Notwithstanding that these studies emphasize the possibilities Skype offers for a language exchange, qualitative analysis notes the need for proper task design in order to maintain high motivation levels [21], [23].

In the case of other platforms such as Adobe connect, similar aims have been sought in different investigations in which the application has been used. In this sense, the need for providing learners with an authentic language experience in which they could put into practice their oral skills was emphasized in different studies using the application [24], [25]. Other queries using this platform such as Cunningham, Fägersten, \& Holmsten's [26] investigated the compensatory mechanisms that took place during the language exchange as well as the kind of conversation taking place in different channels, whether text chat, video or voice. It is worth mentioning Jauregi, Bañados and Morales' [27] research into how video web-communication tools foster the quality of foreign language curricula. This experiment was carried out among students who learnt Spanish as a foreign language and trainee Spanish teachers. Positive findings were reported after the analysis of the sessions, specifically in terms of motivation, use of the language and cultural issues. Other telecommunication platforms such as Polycom [28] or Saba Centra [29] were also investigated to a lesser extent by researchers in the second language learning field.

Regarding the research into WebRTC, Kokane, Singhal, Mukherjee, \& Reddy [30] explored the use of this technology as an interaction platform with $3 \mathrm{D}$ virtual tutors where students could chat as well as listen to their virtual tutors' narrations of articles. At the same time students had access to a timed quiz where they were able to evaluate their own performance. As this technology evolved, new functionalities appeared giving rise to new possibilities for interaction. In this vein, Osipov, Volinsky \& Prasikova [31] made use of this technology to create an online platform called i2istudy ${ }^{\mathrm{TM}}$ that provided predefined scenarios where students could communicate with each other as well as with an instructor. The platform tracks students' progress and instructors are rated by the students subscribed to the platform. This investigation suggested the use of this technology for language learning, as students had the possibility to engage in task-oriented interactions with native speakers. Nevertheless, learners found some pitfalls during its use such as failing to configure the microphone or camera, or using a browser that did not support this technology.

To date, these are the only studies dealing with the use of WebRTC for online learning purposes. Thus, this investigation tries to shed light on this technology and its potential for tele-collaboration and eTandem, as well as explore students' degree of engagement and perceptions about the virtual language exchange. Through an analysis of the information collected during the exchange and the surveys provided to students, we seek to answer the following questions:

- Did the virtual exchange through WebRTC achieve a high degree of engagement in participants?

- Were students' perceptions about the virtual exchange positive? 


\section{$4 \quad$ The Study}

This investigation involved a total of 32 participants from two different universities, 16 from the University of Almeria (Spain) and 16 from Fayetteville University (North Carolina). Participants from the Spanish university were Spanish speakers taking a B1 English as a foreign language course and had 3 teaching contact hours per week. Students from Fayetteville University were American speakers taking a course in Spanish culture and had 4 contact hours per week. The age of the participants ranged from 18 to 27, with 7 male and 25 female participants. The course was given over 4 months from January to April. The web application Jitsi (Figure 2) was introduced in both groups and a timetable with the pairs for the interaction was provided to students.

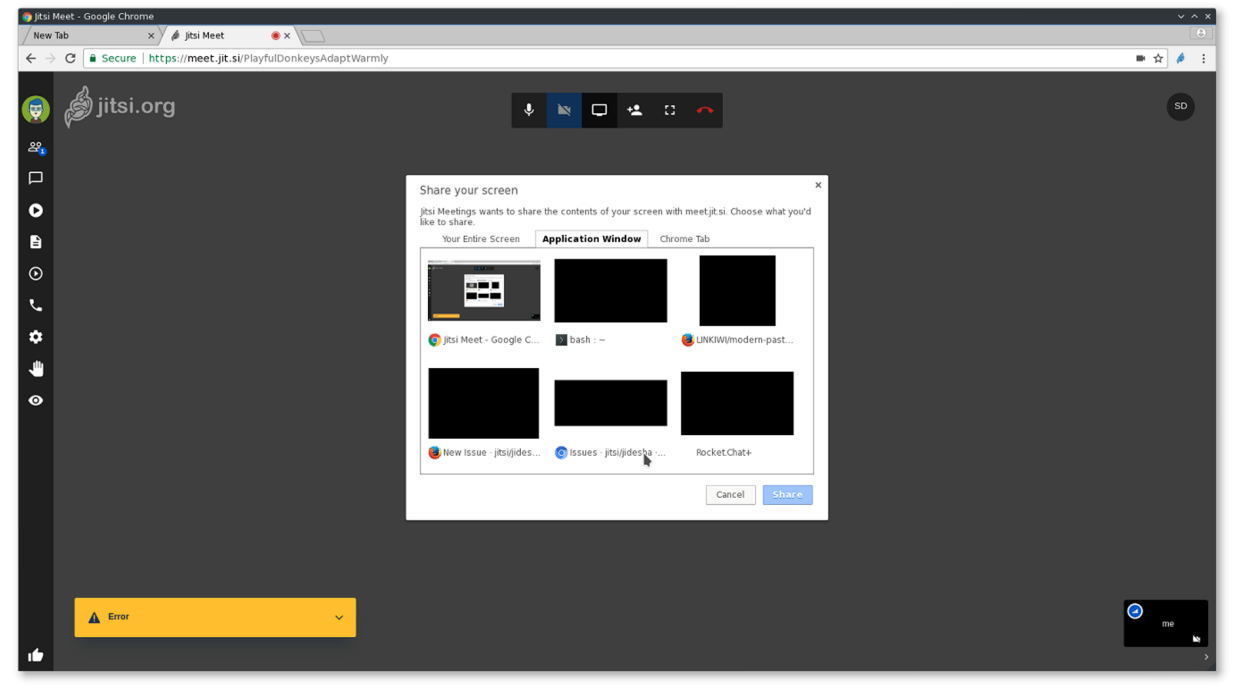

Fig. 2. Jitsi Interface

Participants interacted in dyads twice a week, one from Almería and one from North Carolina, so they could both use their mother tongue and the target language in the exchanges with their respective pairs. Both universities gave permission to the teachers to gather students' email addresses and exchange them with their peers at the other university. Regarding task-design, participants in this virtual exchange are set in a broad spectrum of social constructivism [32]. Co-construction of knowledge as well as reflection play a fundamental role during this process, emphasizing intercultural similarities and differences. The importance of task design has been previously pointed out by researchers in the field [18], [33], [34], thus this investigation followed the criteria of the abovementioned authors to design appropriate interaction tasks: to have language learning potential; to fit learners' needs; to focus on meaning; to be authentic; to have a positive impact on students; and to be practical. Ellis' [34] components of an appropriate task such as goals, input, conditions, procedures and predicted out- 
comes were also taken into consideration when designing the tasks. In order to keep the conversation as authentic as possible and taking into consideration the type of course taken by students in each of the universities, researchers designed two different types of tasks for the interaction. Task 1 consisted of questions and answers about the topics studied in a B1 coursebook. The teacher elaborated a battery of questions every week and two of these questions had to be devised by students. Learners exchanged information about different topics such as food, leisure time or habits among others. Speakers during the interaction were told that in case they noticed any non-target like feature, they should help their peers by giving hints or directly addressing the problem. In task 2 , teacher designed an information gap activity, learners had to get information about the culture of each of the countries, thus both groups of students were given a list of topics they had to prepare in advance in order to answer their peers. Students were asked later in class to report the information obtained during the interaction. In each of the tasks, free conversation was also allowed once these questions were answered. Both teachers set a minimum time of 10 minutes per exchange and there were no limitations to the maximum time. Students had to contact each other every week via mail to arrange a time for the exchange. In order to track students' interaction, a log was created with the start time, end time, day and pair participating in the exchange. Conversations were not recorded to maintain students' privacy, nevertheless they had to send a brief report on the exchange to the teacher after every interaction.

Practical considerations involved: requesting and exchanging students' email addresses in order for the sessions to be clearly organised; guaranteeing all students had a computer or laptop with internet connectivity as well as a built-in microphone, webcam and speakers; minimizing privacy threats [35] introducing common topics that did not include participants' personal details; a 1-hour lesson in both groups was arranged to explain the use of the application and how to start the interaction; supervising the sessions in case any technical problem occurred.

\section{$5 \quad$ Methodology}

The research design was considered at the beginning of the activity. A mixed methods approach [36] was followed as quantitative and qualitative data were analysed in order to measure students' degree of engagement in the application. Indications by Henrie, Halverson, \& Graham [37], experts in measuring students' engagement in technology-mediated learning, were followed. In this sense, the technology category index (TACI) [38] was adapted in order to assess students' pre-existing knowledge about using this technology (Appendix A). This survey was given to participants at the beginning of the investigation in order to minimize possible threats to validity, such as the impact that the relation with the learning subject or students' ability to use technology could have on the time students spent on the activity. During the activity, a log registering the time spent by students in each of the exchanges was used to report students' degree of engagement throughout the investigation. This log was also used by both teachers to keep control of the topics and questions used 
throughout the interaction, as well as to understand students' behaviour in the virtual exchange. Finally, Henrie's [39] longitudinal scale to measure students' engagement (Appendix B) was used at the end of the investigation as our aim was to measure students' engagement at an activity level. This scale measured the three different types of engagement described by Fredricks, Blumenfeld and Paris [40]: behavioral, emotional and cognitive engagement. According to the abovementioned author, behavioral engagement encompasses those behaviors related with academic success such as an active participation, high attendance levels or homework completion. The second type of engagement, in this case emotional, deals with students' feelings about the learning experience such as interest, boredom, apathy and frustration. Finally, cognitive engagement refers to students' conscious efforts to understand the explanations, including self-regulation processes and metacognitive behaviors.

Regarding students' perception about the telecollaboration project, an end-ofcourse survey (Appendix C) was provided to students in order to explore their perspectives about the use of WebRTC, advantages and pitfalls. This survey was based on Bañados, Jauregi and Morales' [27] video-web telecollaboration project and was adapted to answer particular questions raised in this investigation.

The scale as well as both surveys were analysed quantitatively except for the openended items that allowed the researcher to better understand students' opinions about the exchange. Internal threats to validity in mixed methods research [41] were minimized as the data collection instruments had been corroborated in previous studies [27], [37], [39] and the researchers followed the indications of experts in each of the areas under analysis. Nevertheless, limitations regarding the number of participants may put in question the generalizability of the results.

\section{$6 \quad$ Results}

Firstly, the results of the pre-existing knowledge survey are presented in Figures 2 and 3 in order to observe initial differences among the students participating in the telecollaboration project. These differences were observed as researchers in the field of technology-mediated learning pointed out a strong correlation between students' pre-existing knowledge of the subject as well as their ability to use technology, and the amount of time participants spend on the virtual exchange [37].

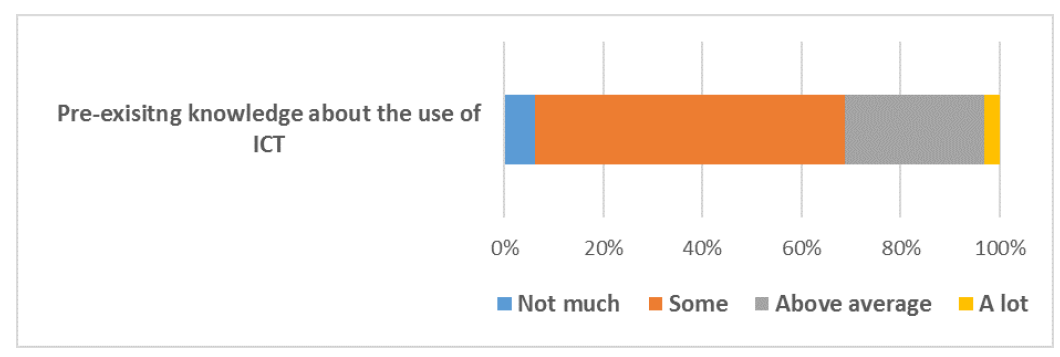

Fig. 3. Pre-existing knowledge survey on ICT 
Items regarding students' pre-existing knowledge were grouped in Figure 3. Results indicated that a bit more than a half of the participants had some knowledge on the use of ICT, and another 40 per cent pointed out that their knowledge was high or very high. These results emphasize that learners in both groups were familiar with technologies, use of the internet and thus may not have many problems adapting to this virtual exchange. On the other hand, figures about students' experience on eTandem, virtual exchanges and WebRTC indicated that most of the students had no previous experience with 90 per cent of participants answering "No" to the aforementioned items, emphasizing that for the majority of the students, it was the first time they had carried out this kind of interaction. In this respect, we can be assured that students' engagement during the interaction was not biased by their pre-existing knowledge as regards previous experiences in this type of activity.

A tracking of the interaction was also used to analyse the time spend by participants in each of the exchanges. Statistics indicated that the average amount of time per exchange was 41,9 minutes, highlighting a high degree of engagement as students were not given a maximum time to speak with their peers. Moreover, this average time may suggest that when students were involved in social interaction, they did not feel as if they were doing out-of-class work but simply meeting new peers while using the L2. The maximum amount of time spent by a participant was 80 minutes whereas the minimum was 8 minutes. Figure 4 shows a graphical representation of the range of time spent by each of the Spanish participants in the exchange with their American peers.

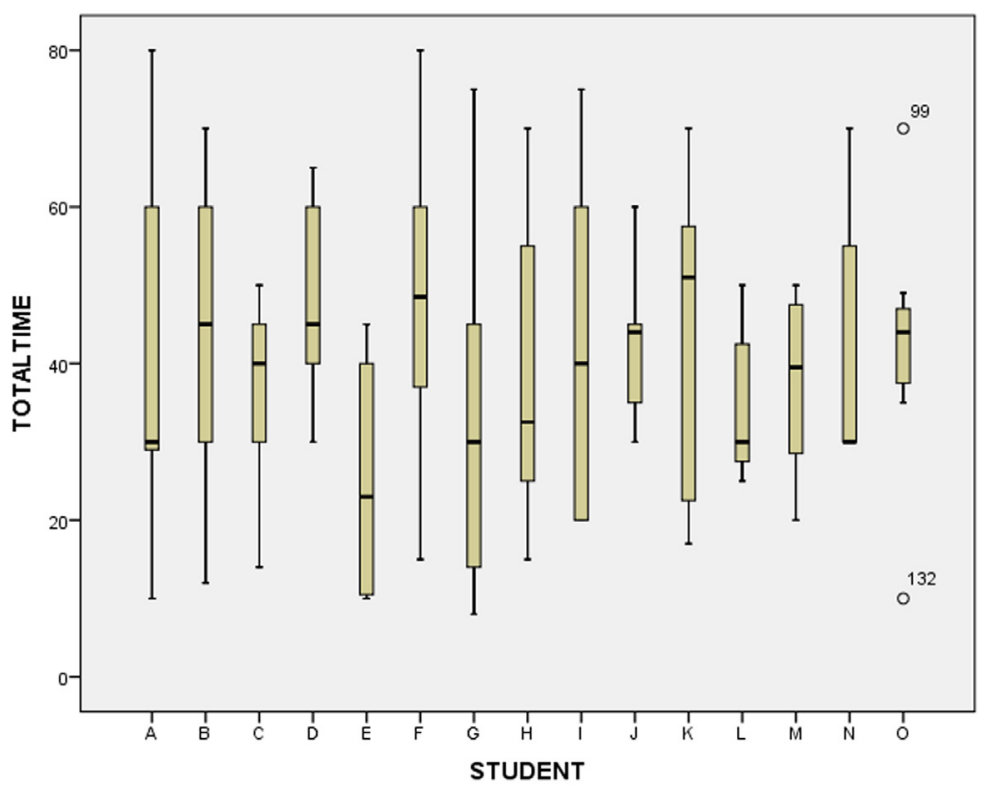

Fig. 4. Range of time used per student in the exchange 
The log also registered the start time and end time, giving the researchers an idea of the best times for students to interact. As participants needed to arrange a time with their peers and there was a 5-hour time difference, conversations normally took place in the afternoon or evening (Central European Summer Time) for Spanish participants and in the morning or midday for the American students (Eastern Time). The first week of research, this situation gave rise to misunderstanding between peers which led to participants complaining because their peers were not online. Nevertheless, this situation only took place at the very beginning of the activity.

In order to continue measuring the engagement of the students in the exchange, the results of the engagement scale provided at the end of the course are presented in the following section. Frequencies, inferential statistics and effect size were used to analyse and report the information collected in the scale, offering a wider picture of students' engagement at the end of the course. Firstly, emotional engagement data was evaluated taking into consideration the items 1-4 in the engagement scale (Appendix B). The results regarding the degree of enjoyment and interest of students noted the high degree of acceptance earned by the exchange as well as the technology used. In this sense, the one-sample Wilcoxon ranked test indicated that the median was significantly different from $2.5, Z=5.18, p<0.01$, with a very strong effect size $(r=.91)$ in the case of students' enjoyment of WebRTC. Similarly, inferential statistics measuring students' interest found significant differences $(Z=5.44, p<0.01, r=.96)$, with the majority of respondents indicating that their interest increased throughout the activity. Participants were also asked if they wished they had been doing something else instead of participating in the exchange and their responses indicated a high degree of engagement $(\mathrm{Z}=4.29, p<0.01, \mathrm{p}=.74)$ as shown in the significance value and effect size, with a high percentage of students indicating "not at all". Further measures of emotional engagement such as students' degree of excitement also presented high frequencies with an 84 per cent of students indicating "Very excited" and 16 per cent marking "Excited".

The second part of the scale tackled students' cognitive engagement and a sevenpoint semantic differential response scale was used, with the positive end indicating an aspect of engagement and the negative end indicating disengagement. Measures of cognitive engagement are presented in Table 1.

Table 1. Students' cognitive engagement throughout the exchange

\begin{tabular}{|l|c|c|c|c|c|l|}
\hline & Max & Medium & Neutral & Medium & Min & \\
\hline Active & $0 \%$ & $94 \%$ & $6 \%$ & $0 \%$ & $0 \%$ & Passive \\
\hline Focused & $91 \%$ & $9 \%$ & $0 \%$ & $0 \%$ & $0 \%$ & Distracted \\
\hline
\end{tabular}

As shown in the frequency of students' responses as well as in the open-ended questions of the end-of-course survey that will be further discussed in this section, participants highlighted that they were very excited, particularly, before the start of the conversation as they were going to meet someone for the first time through the web. In this sense, they also pointed out their high degree of attention to the language of their peers, as they at times, struggle to understand certain pronunciations and asked for repetition to understand the message. 
Students' perception of their degree of concentration was also measured with significant results $(\mathrm{Z}=5.25, p<0.01, \mathrm{r}=.92)$ which correlate with the previous finding regarding how focused students were during the interaction.

Following the transactional model of student engagement [39], [42], [43], in which the learning environment as well as learners' characteristics are a fundamental elements of students' engagement, the type of learners in the activity were evaluated through the items in the longitudinal scale. In this respect, items 8 to 11 and item 13 of the scale evaluated students' positive attitude towards the course as well as their degree of confidence in passing the module. These items were analysed and grouped in order to have a visual representation of the abovementioned items and are presented in Figure 4.

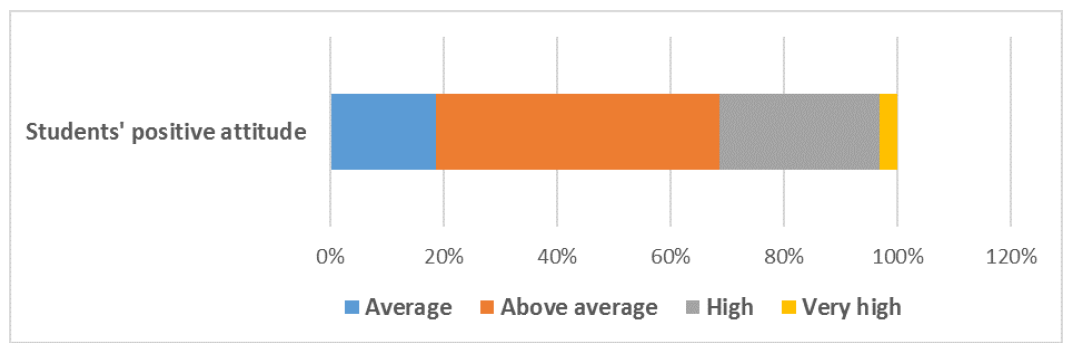

Fig. 5. Students' positive attitude towards the course

As presented in Figure 5, more than a half of the students participating in the interaction responded that their positive attitude was "above average", hence this predisposition towards the module may have contributed to increase students' degree of engagement. Nevertheless, participants did not present maximum scores in a high proportion with regard to this parameter, thus engagement cannot be directly justified because of students' attitude towards the module. In order to take into consideration students' attitude from a different angle, items 12 and 14 evaluated students' negative attitude towards the course and the module. This parameter correlates with the previous one as students' did not specifically present a negative attitude towards the course as presented in Figure 6, nevertheless most of responses were "below average" or "average", emphasizing that their mood was positive but quite neutral at the same time.

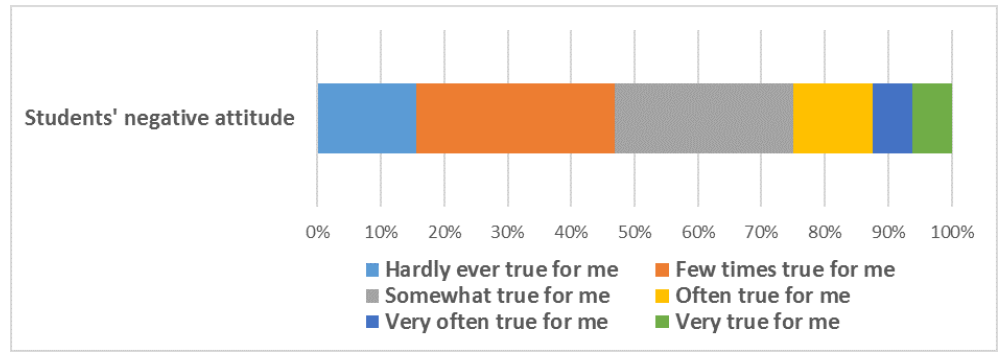

Fig. 6. Students' negative attitude towards the course 
Further measures or possible explanations of students' engagement were taken into consideration in the scale such as the importance and interest of students towards the subject matter. Items 15 to 18 dealt with this parameter and were grouped for analysis purposes, in order to evaluate to what extent the content of the course was significant and interesting for students participating in the interaction as presented in Figure 7.

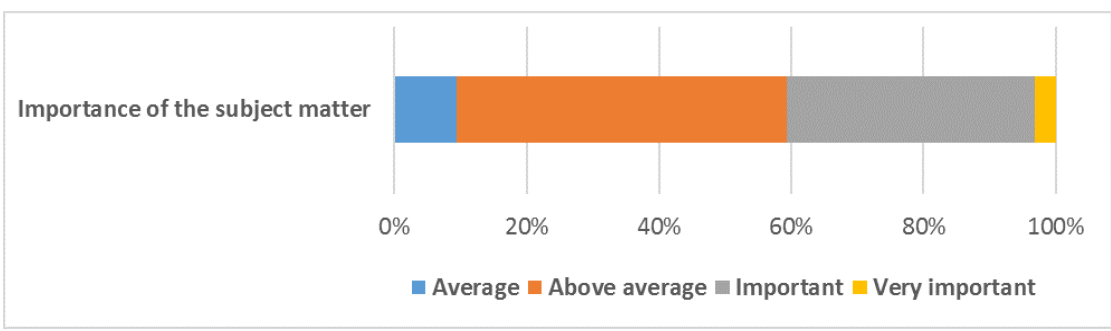

Fig. 7. Students' perception of the importance of the subject matter

Most of students marked "above average" or "important", highlighting that participants gave careful attention to the contents of the course and thus, contributed to a higher degree of engagement. In this sense, as abovementioned in the study section, task design became a fundamental part of the telecollaboration project and teachers worked thoroughly to address the contents of the course in the virtual exchange. Task were specifically designed to meet the requirements, contents and syllabuses of each course.

Students' mood towards learning and passing modules was also observed in items 21-28, particularly their degree of apathy was addressed in items 21 to 24 as well as their eagerness to learn in items $25-28$. With the aim of observing students' characteristics in these aspects, we grouped the responses of participants and the results can be observed in Table 2.

Table 2. Students' degree of apathy and eagerness to learn

\begin{tabular}{|l|c|c|}
\hline & Students' apathy & Student's eagerness to learn \\
\hline Never or only rarely true for me & $0 \%$ & $3 \%$ \\
\hline Sometimes true for me & $16 \%$ & $34 \%$ \\
\hline True for me about half the time & $59 \%$ & $53 \%$ \\
\hline Often true for me & $16 \%$ & $9 \%$ \\
\hline Always or almost always true for me & $0 \%$ & $0 \%$ \\
\hline
\end{tabular}

As observed in Table 2, similar percentages were found in each of the categories, particularly those indicating that about a half of the participants felt apathy or eagerness to learn to a certain extent (True for me about half the time) in particular learning situations. Notwithstanding these percentages, the tendency of participants indicated that they were eager to learn in more situations than they felt apathetic as reflected in the abovementioned figures with a 34 per cent of students marking the option number 4 in the Likert scale.

Thus, answering the first research question in this investigation, findings confirmed that the telecollaboration project through WebRTC achieved a high degree of en- 
gagement of students participating in the activity, whether emotional, behavioral or cognitive.

The end-of-course survey considered students' perceptions of WebRTC, advantages and pitfalls. Close and open-ended items were incorporated into the survey to have a wider perspective of students' opinion as regards the virtual exchange. Inferential statistics, specifically, the one-sample Wilcoxon ranked test were used to explored significant differences in the answers to the close-ended items provided by participants. In this sense, in every item from 1 to 7 , the median was significantly different from 2.5, and factors such as the value of the video-web project $(Z=$ $4.85, p<0.01, r=.88)$, use of Jitsi $(Z=4.47, p<0.01, r=.81)$, functioning of the virtual environment $(Z=4.70, p<0.01, r=.85)$, usefulness of the interaction $(Z=$ 4.92, $p<0.01, r=.89)$ and students' interest $(Z=4.90, p<0.01, r=.89)$ highlighted a very positive perception of students towards the experiment carried out.

On the other hand, open-ended as well as yes/ no items gave us an insight into some of the particular advantages and pitfalls encountered during the project. In this sense, as regards the first open-ended item of the perception scale regarding differences between communication face-to-face and through the virtual platform, participants emphasized the telecommunication tool as a good alternative due to the possibility of speaking with another person anytime and anywhere as well as showing your peers your life in a different country. These findings had already been found in other tele-collaboration projects such Levak \& Son's [17] or Vinagre's [44] who emphasized the power of telecommunication to develop intercultural competence from different places. On the contrary, yes/no items in the scale regarding students' preferences between both environments indicated that the majority of students $(73 \%)$ preferred face-to-face communication to online interaction.

As regards the positive aspects found by students during the virtual exchange, many answers dealt with the possibility of improving their second language while meeting new people, in line with telecollaboration studies [45], [46] emphasizing the importance of social interaction to develop the L2. Further positive aspects indicated the ease of using the application without having to download any software or access a personal account. On the other hand, negative aspects, also considered in the openended items of the scale, highlighted different factors such as the difficulty to arrange a time because of the time difference, or the quality of the internet connection that has been a constant pitfall in telecollaboration studies to date [17], [47], [48]. Students also pointed out possible improvements to the project, particularly in the way to arrange the time of the exchanges, as they had to contact their peers through email but, due to its asynchronous character, it sometimes gave rise to delays in the scheduling of the sessions. In this sense, students suggested creating a chat group with their peers, an idea that was quickly accepted by the teachers involved in the telecollaboration project.

Further close-ended items dealing with the integration of the telecollaboration project in the course as well as whether participants would recommend it to peers found very positive results with students in both classes responding unanimously "Yes" to both items. Thus, answering the second research question in this investigation, students' perceptions of the telecollaboration project through WebRTC were significant- 
ly positive except for the abovementioned pitfalls that, in some cases, practitioners could not avoid.

\section{Conclusion}

The experience of this telecollaboration project through WebRTC has led to exploring the potential of this videoconferencing tool to foster students' engagement at an activity level. In this sense, the use of web real time communication through the browser allowed researchers to explore students' degree of engagement at an emotional, behavioral and cognitive level. The results of the pre-existing knowledge survey as well as those from the engagement scale indicated a significant effect of the telecollaboration project on students' engagement, which has been linked to relevant educational outcomes, such as students' persistence in learning [40], [49], satisfaction [50] and academic achievement [51]. Nevertheless, limitations as regards the number of students participating in the telecollaboration project may affect the results of this investigation, thus further research analyzing students' engagement in telecollaboration projects is sought.

The project allowed practitioners in the investigation to create opportunities for language interaction, creating a naturalistic conversation that, as argued by researchers in the field [52], [53], could lead to an appropriate preparation for real face-to-face situations. At the same time, students' perceptions of the use of WebRTC were observed in order to evaluate whether the use of this protocol could give rise to a change in the paradigm of traditional videoconferencing systems in use for telecollaboration. In this sense, previous findings of telecollaboration projects such as the development of intercultural competence or language communicative skills [27], [47] were also regarded through the use of WebRTC. The use of this system earned a high acceptance by students who quickly adapted to the platform, making use of the advantages of these types of telecommunication offers over already existing videoconferencing tools such as P2P connectivity, web-based technology, open source protocol or free of charge use among others. Although some of the pitfalls present in telecollaboration studies were still present through the use of this technology such as internet connectivity or time zones, the ease of accessing the videoconferencing system through the web avoided software installation and user privacy issues found in previous studies [6], [17]. Furthermore, due to its inexpensive cost, higher institutions and practitioners could benefit from its use, connecting classrooms, increasing intercultural competence and expanding students' in-class time, thus in line with students' perceptions in this research, steps need to be taken regarding its integration in the syllabus of language learning programs. In this sense, this technology becomes an open educational resource (OER) at the service of practitioners as well as students willing to collaborate to generate cross-cultural knowledge and foster students' opportunities for language practice and feedback. 


\section{References}

[1] M. Dooly, "Telecollaboration," in The Handbook of Technology and Second Language Teaching and Learning, Wiley-Blackwell, 2017, pp. 169-183. https://doi.org/10.1002/9781118914069.ch12

[2] B. Bauer, . L. deBenedette, G. Furstenberg, S. Levet and S. Waryn, "The Cultura Project," in Internet-mediated Intercultural Foreign Language Education, Boston, Heinle \& Heinle, 2006, pp. 31-36.

[3] D. Perez-Hernandez, "Chronicle.com," 5 May 2014. [Online]. Available: https://www.chronicle.com/article/Technology-Provides/146369. [Accessed 1 September 2018].

[4] M. Dooly and R. O'Dowd, Researching Online Foreign Language Interaction and Exchange, Bern, Switzerland: Peter Lang, 2012. https://doi.org/10.3726/978-3-0351-04141

[5] U. Stickler and T. Lewis, "Collaborative language learning strategies in an email tandem exchange," in Language Learning Strategies in Independent Settings, vol. 33, Bristol, UK, Multilingual Matters, 2008, p. 237-261. https://doi.org/10.21832/9781847690999-015

[6] J. Tian and Y. Wang, "Taking language learning outside the classroom: learners' perspectives of eTandem learning via Skype," Innovation in Language Learning and Teaching, vol. 4, no. 3, pp. 181-197, 2010. https://doi.org/10.1080/17501229.2010.513443

[7] A. Barron and E. Black, "Constructing small talk in learner-native speaker voice-based telecollaboration: A focus on topic management and backchanneling," System, vol. 48, pp. 112-128, 2015. https://doi.org/10.1016/j.system.2014.09.009

[8] D. Ito, M. Niibori and M. Kamada, "A real-time web-cast system for classes in the byod style," in Network-Based Information Systems (NBiS), 2016 19th International Conference on, Ostrava, Czech Republic, 2016. https://doi.org/10.1109/NBiS.2016.33

[9] "Is WebRTC ready yet," [Online]. Available: http://iswebrtcreadyyet.com/.. [Accessed 8 February 2018].

[10] A. Karabulut and A. Correia, "Skype, Elluminate, Adobe Connect, Ivisit: A comparison of web-based video conferencing systems for learning and teaching," in Society for information technology $\mid \&$ teacher education international conference, Chesapeake, VA, 2008.

[11] S. Schullo, A. Hilbelink, M. Venable and A. E. Barron, "Selecting a virtual classroom system: Elluminate live vs. Macromedia breeze (adobe acrobat connect professional)," MERLOT Journal of Online Learning and Teaching, vol. 3, no. 4, pp. 331-345, 2007.

[12] R. L. Moore and B. P. Fodrey, "Distance Education and Technology Infrastructure: Strategies and Opportunities," in Leading and Managing e-Learning, Springer, 2018, pp. $87-100$.

[13] M. d. C. B. Lopez, M. E. Ortiz and C. Allen, "Using mobile devices and the Adobe Connect web conferencing tool in the assessment of EFL student teacher performance," in Critical CALL--Proceedings of the 2015 EUROCALL Conference, Padova, Italy, 2015.

[14] M. Bower, B. Dalgarno, G. E. Kennedy, M. J. Lee and J. Kenney, "Design and implementation factors in blended synchronous learning environments: Outcomes from a cross-case analysis," Computers \& Education, vol. 86, pp. 1-17, 2015. https://doi.org/10.1016/i.compedu.2015.03.006

[15] Y. Akbaba and F. Baskan, "How to merge courses via Skype ${ }^{\mathrm{TM}}$ ? Lessons from an International Blended Learning Project," Research in Learning Technology, vol. 25, 2017.

[16] N. Austin, R. Hampel and A. Kukulska-Hulme, "Video conferencing and multimodal expression of voice: Children's conversations using Skype for second language 
development in a telecollaborative setting," System, vol. 64, pp. 87-103, 2017. https://doi.org/10.1016/j.system.2016.12.003

[17] N. Levak and J.-B. Son, "Facilitating second language learners' listening comprehension with Second Life and Skype," ReCALL, vol. 29, no. 2, pp. 200-218, 2017. https://doi.org/10.1017/S0958344016000215

[18] K. Jauregi, R. De Graaff, H. van den Bergh and M. Kriz, "Native/non-native speaker interactions through video-web communication: A clue for enhancing motivation?," Computer Assisted Language Learning, vol. 25, no. 1, pp. 1-19, 2012. https://doi.org/10.1080/09588221.2011.582587

[19] L. Szedmina and R. Pinter, "Experiences from using Skype in language teaching," in Intelligent Systems and Informatics (SISY), 2010 8th International Symposium on, 2010. https://doi.org/10.1109/SISY.2010.5647340

[20] Y. Romaña Correa, "Skype ${ }^{\mathrm{TM}}$ conference calls: A way to promote speaking skills in the teaching and learning of English," Profile Issues in TeachersProfessional Development, vol. 17, no. 1, pp. 143-156, 2015. https://doi.org/10.15446/profile.v17n1.41856

[21] N. M. Terhune, "Language learning going global: linking teachers and learners via commercial Skype-based CMC," Computer Assisted Language Learning, vol. 29, no. 6, pp. 1071-1089, 2016. https://doi.org/10.1080/09588221.2015.1061020

[22] R. Godwing-Jones, "Skype and podcasting: Disruptive Technologies for language learning," Language Learning and Technology, vol. 9, no. 3, pp. 9-12, 2005.

[23] T. Mullen, C. Appel and T. Shanklin, "Skype-based tandem language learning and web 2.0," in Handbook of research on Web 2.0 and second language learning, Hershey, PA, Igi Global, 2009, pp. 101-118. https://doi.org/10.4018/978-1-60566-190-2.ch006

[24] E. Longhi and C. Angelini, "How to Use Adobe Connect for Oral Language Practice in International Virtual Classes," in ICT for Language Learning International Conference, Florence, Italy, 2015.

[25] M. Mehrabi and S. Homapour, "The effect of the substrate type in virtual concurrent classes on the oral comprehension of the Iranian language learners: the case of Adobe Connect Platform and Skype Software," Language Related Research, vol. 9, no. 2, pp. 251-276, 2018.

[26] U. M. Cunningham, K. B. Fägersten and E. Holmsten, "' Can you hear me, Hanoi?" Compensatory mechanisms employed in synchronous net-based English language learning," The International Review of Research in Open and Distributed Learning, vol. 11, no. 1, pp. 161-177, 2010. https://doi.org/10.19173/irrodl.v11i1.774

[27] K. Jauregi and E. Bañados, "Virtual interaction through video-web communication: A step towards enriching and internationalizing language learning programs," $\operatorname{ReCALL}$, vol. 20, no. 2, pp. 183-207, 2008. https://doi.org/10.1017/S0958344008000529

[28] M. Chakraborty and S. Victor, "Do's and don'ts of simultaneous instruction to on-campus and distance students via videoconferencing," Journal of library administration, vol. 41, no. 1-2, pp. 97-112, 2004. https://doi.org/10.1300/J111v41n01_09

[29] C. White, P. Ramirez, J. Smith and L. Plorowski, "Simultaneous delivery of a F2F course to on-campus and remote off-campus students," TechTrends, vol. 54, no. 4, pp. 34-40, 2010. https://doi.org/10.1007/s11528-010-0418-z

[30] A. Kokane, H. Singhal, S. Mukherjee and G. Reddy, "Effective e-learning using 3D virtual tutors and WebRTC based multimedia chat," in Recent Trends in Information Technology (ICRTIT), 2014 International Conference on, 2014. https://doi.org/10.1 109/ICRTIT.2014.6996209 
[31] I. V. Osipov, A. A. Volinsky and A. Y. Prasikova, "E-learning collaborative system for practicing foreign languages with native speakers," International Journal of Advanced Computer Science and Applications, vol. 7, no. 3, 2016.

[32] L. Vygotsky, "Interaction between learning and development," Readings on the development of children, vol. 23, no. 3, pp. 34-41, 1978.

[33] C. A. Chapelle, English language learning and technology: Lectures on applied linguistics in the age of information and communication technology, John Benjamins Publishing, 2003. https://doi.org/10.1075/11lt.7

[34] R. Ellis, Task-based language learning and teaching, Oxford University Press, 2003.

[35] D. M. Boyd and N. B. Ellison, "Social network sites: Definition, history, and scholarship," Journal of computer-mediated Communication, vol. 13, no. 1, pp. 210-230, 2007. https://doi.org/10.1111/j.1083-6101.2007.00393.x

[36] J. W. Creswell and V. L. P. Clark, Designing and conducting mixed methods research, Sage publications, 2017.

[37] C. R. Henrie, R. Bodily, K. C. Manwaring and C. R. Graham, "Exploring intensive longitudinal measures of student engagement in blended learning," The International Review of Research in Open and Distributed Learning, vol. 16, no. 3, 2015. https://doi.org/10.19173/irrodl.v16i3.2015

[38] C. A. Dugas, Adopter characteristics and teaching styles of faculty adopters and nonadopters of a course management system, Indiana State University, 2005.

[39] C. Henrie, R. Larsen, K. Manwaring, L. Halverson and C. Graham, "Validation of a longitudinal activity-level measure of student engagement," Contemporary Educational Psychology, 2016.

[40] J. A. Fredricks, P. C. Blumenfeld and A. H. Paris, "School engagement: Potential of the concept, state of the evidence," Review of educational research, vol. 74, no. 1, pp. 59-109, 2004. https://doi.org/10.3102/00346543074001059

[41] R. B. Johnson, A. J. Onwuegbuzie and L. A. Turner, "Toward a definition of mixed methods research," Journal of mixed methods research, vol. 1, no. 2, pp. 112-133, 2007. https://doi.org/10.1177/1558689806298224

[42] J. Eccles and M.-T. Wang, "Part I commentary: So what is student engagement anyway?," in Handbook of research on student engagement, Springer, 2012, pp. 133-145.

[43] M. A. Lawson and H. A. Lawson, "New conceptual frameworks for student engagement research, policy, and practice," Review of Educational Research, vol. 83, no. 3, pp. 432479, 2013. https://doi.org/10.3102/0034654313480891

[44] M. Vinagre, "El aprendizaje intercultural en entornos virtuales de colaboración," Revista española de lingüística aplicada, no. 23, pp. 297-320, 2010.

[45] L. Lee, "Using Telecollaboration 2.0 to Build Intercultural Communicative Competence: A Spanish-American Exchange," in Cross-Cultural Perspectives on Technology-Enhanced Language Learning, IGI Global, 2018, pp. 303-321. https://doi.org/10.4018/978-1-52255463-9.ch017

[46] T. Lewis, B. O'Rourke and M. Dooly, "Online Intercultural Exchange," Innovation in Language Learning and Teaching, vol. 10, no. 1, 2016.

[47] A. Nicolaou and A. Sevilla-Pavón, "Exploring Telecollaboration through the Lens of University Students: A Spanish-Cypriot Telecollaborative Exchange," in New directions in telecollaborative research and practice: selected papers from the second conference on telecollaboration in higher education, Research-publishing.net, 2016, p. 113.

[48] J. I. Liontas, The TESOL Encyclopedia of English Language Teaching, 2018. https://doi.org/10.1002/9781118784235 
[49] G. D. Kuh, T. M. Cruce, R. Shoup, J. Kinzie and R. M. Gonyea, "Unmasking the effects of student engagement on first-year college grades and persistence," The journal of higher education, vol. 79, no. 5, pp. 540-563, 2008. https://doi.org/10.10 80/00221546.2008.11772116

[50] A. D. Lewis, E. S. Huebner, P. S. Malone and R. F. Valois, "Life satisfaction and student engagement in adolescents," Journal of Youth and Adolescence, vol. 40, no. 3, pp. 249262, 2011. https://doi.org/10.1007/s10964-010-9517-6

[51] M. R. Reyes, M. A. Brackett, S. E. Rivers, M. White and P. Salovey, "Classroom emotional climate, student engagement, and academic achievement," Journal of educational psychology, vol. 104, no. 3, p. 700, 2012. https://doi.org/10.1037/a0027268

[52] V. Tudini, "Form-focused social repertoires in an online language learning partnership," Journal of pragmatics, vol. 50, no. 1, pp. 187-202, 2013. https://doi.org/10.1016/j. pragma.2012.12.005

[53] P. D. Ware and C. Kramsch, "Toward an intercultural stance: Teaching German and English through telecollaboration," The Modern Language Journal, vol. 89, no. 2, pp. 190205, 2005. https://doi.org/10.1111/j.1540-4781.2005.00274.x

\section{Authors}

Alberto Andujar is lecturer at the English department of the University of Almería, Spain. He specializes into second language learning and technology, computerassisted language and mobile-assisted language learning. He is also author of different international research articles in these fields and is currently developing projects into the use of virtual reality for second language learning (alberto.andujar@ual.es).

Cristóbal Medina-López is a Ph.D. student at the University of Almería, where he is a member of the Supercomputing-Algorithms Research Group. His research interests include P2P networks, network security, and multimedia streaming over the Internet (cristobalmedina@ual.es).

Article submitted 2018-09-28. Resubmitted 2018-12-05. Final acceptance 2018-12-05. Final version published as submitted by the authors. 